\title{
Ag Ion Decoration for Surface Modifications of Multi-walled Carbon Nanotubes
}

\author{
Dilermando N. Travessa ${ }^{a *}$, Fábio S. da Silva ${ }^{\mathrm{b}}$, Fernando H. Cristovan ${ }^{\mathrm{a}}$,
}

\author{
Alberto M. Jorge Jr. ${ }^{c}$, Kátia Regina Cardoso ${ }^{a}$
}

anstituto de Ciência e Tecnologia, Universidade Federal de São Paulo - UNIFESP,

Rua Talim, 330, CEP 12231-280, São José dos Campos, SP, Brazil

${ }^{\mathrm{b}}$ Empresa Brasileira de Aeronáutica S.A. - Embraer, Av. Brigadeiro Faria Lima, 2170,

CEP 12227-901, São José dos Campos, SP, Brazil

'Departamento de Engenharia de Materiais, Universidade Federal de São Carlos - UFSCar, Rod. Washington Luís, Km 235, São Carlos, SP, Brazil

Received: August 26, 2013; Revised: January 30, 2014

\begin{abstract}
The production of high performance metal matrix composites depends on a proper design of the surface of the reinforcing phase, ensuring a good contact with a metal phase. In the present work, two Ag decorating procedures to modify the surface of multi-walled carbon nanotubes (MWCNT) were evaluated for further production of aluminum matrix composites. The procedures consisted in a two steps route based on acid oxidation of carbon nanotubes (CNT) followed by suspension in an $\mathrm{Ag}$ ion solution; and a single step route, based on the effect of $\mathrm{n}$-dimethylformamide (DMF) as an activation agent of CNT surface, in presence of $\mathrm{Ag}$ ions. Transmission and scanning-transmission electron microscopy, Raman and Fourier-transformed infrared spectroscopy were employed in order to characterize the results. The two steps route resulted in Ag nano-particles homogeneously deposited over the CNT surface. The mechanism for the deposition is based on carboxyl and probably hydroxyl functional groups formed in the first step, acting as nucleation sites for Ag precipitation in the second step. The single step route resulted in the formation of sub-micrometric Ag particles heterogeneously mixed to CNT bundles.
\end{abstract}

Keywords: carbon nanotubes, $A g$ decoration, acid refluxing, surface activation

\section{Introduction}

Among the known structural forms of carbon, three of them are receiving special attention in the last two decades: fullerenes, nanotubes and graphenes ${ }^{1}$. Carbon nanotubes (CNT), having a sp² type hybridization in a tridimensional atomic array, show some of physical, chemical and mechanical properties that are intermediate to graphite and diamond, and are unique among other materials. Very high thermal conductivity, high thermal and chemical stability, low friction and an electric behavior that varies from semi to high conductivity (depending on their structure) are examples of such interesting properties ${ }^{1,2}$. Particularly, an elastic modulus of the order of 1,0 to $1,8 \mathrm{TPa}$, a tensile strength ranging from 50 to $150 \mathrm{GPa}$ and a density ranging from 1,2 to $2,2 \mathrm{~g} / \mathrm{cm}^{3[1-3]}$ make the CNT very attractive as a reinforcing phase in light and ductile metal matrix composites.

In order to take a technological advantage of the interesting properties of CNT, engineering products have to be designed and the production of metal, ceramic or polymers composites reinforced by CNT is one of the promising strategies to produce useful goods. The production of high quality composite materials, in turn, depends on a homogeneous dispersion of the reinforcement

*e-mail: dilermando.travessa@unifesp.br phase into the matrix. Furthermore, a good contact at the matrix/reinforcement interface has to be achieved. CNT, due to their small size and high surface area tend to agglomerate, making the composite processing very challenge. It is known, for instance that graphene has low wettability by molten aluminum ${ }^{4}$, suggesting a poor interaction at the interface.

Recently, several attempts have been performed in order to modify the CNT surface, for several purposes: catalytic applications, nano-sized electronic devices, drugs and molecules delivering systems, bactericide action, biosensors, biological images, energy storage, water cleaning, and to improve the dispersion of CNT into several matrix systems $^{5-17}$. Graphene surface has also been modified for similar reasons ${ }^{18,19}$. The deposition of metallic atoms on the surface, by chemical routes, is one of the most common processes for surface modification of CNT. It is usually performed in steps: the functionalization that consists in an acid treatment, in order to create functional carboxylic groups chemically attached to the CNT surface ${ }^{6-9,13-15,17,20,21}$, and the decoration that consists usually in obtaining a suspension of the functionalized CNT into a water soluble metallic salt $7,9,14,16,22$. The metallic salt is responsible for supplying the ions that will bond to the carboxylic groups, forming deposits on the CNT surface. 
The functionalization and decoration of CNT with metallic ions is mainly performed in order to change their physical, chemical and electronic properties, creating specific functions for them. It can be also performed to improve the dispersion of CNT in metal matrix composites (CNT-MMC), creating repulsive charges that diminish their tendency to agglomerate ${ }^{15,23}$. Another very promising benefit of metallic decoration of CNT that hasn't been explored is the improvement of the contact (adhesion) at the interface with the metallic matrix in CNT-MMC. A better interaction can enhance the load transfer process from the matrix to the reinforcement phase, resulting in high strength composites. In this context, the present work deals with Ag decoration of MWCNT for further production of CNT reinforced aluminum matrix composites (CNT-AMC). The use of Ag is very interesting due to the microstructural benefits that it produces in aluminum alloys. Particularly in Al-Mg-Si systems like the AA6061 aluminum alloy, Ag additions can improve the strength and ductility, and accelerates the agehardening response $\mathrm{e}^{24}$. Two decorating processes, existing in the literature, were evaluated: a modified two steps process, based on CNT acid refluxing followed by the suspension in an $\mathrm{AgNO}_{3}$ solution ${ }^{9}$, and a single step process, based on the activation of CNT surface by n-dimethylformamide (DMF) in an $\mathrm{AgNO}_{3}$ solution ${ }^{16}$. Both processes are relatively simple, as no complicated reagents or sophisticated equipments are necessary. Furthermore, a large amount of CNT can be processed at once. Fourier transformed-infrared (FT-IR) and Raman spectroscopy, as well as transmission, scanningtransmission and high resolution transmission electron microscopy (TEM, STEM and HRTEM), were employed in order to characterize MWCNT before and after decoration.

\section{Experimental Procedures}

\subsection{Materials}

In the present work, the following materials have been used:

- MWCNT supplied by Bayer Materials Science (Baytubes C150P), in the form of bundles;

- Nitric acid $\left(\mathrm{HNO}_{3}\right)$, analytical grade;

- Silver nitrate $\left(\mathrm{AgNO}_{3}\right)$, analytical grade;

- n-dimethylformamide (DMF), analytical grade;

- Sodium dodecyl sulfate (SDS), analytical grade;

- De-ionized water.

\subsection{Two steps Ag decoration}

Typically, $0.2 \mathrm{~g}$ of MWCNT were de-agglomerated in an agate mortar and subsequently dispersed into $25 \mathrm{ml}$ of a $\mathrm{HNO}_{3}$ solution (7 molar), under ultrasonic agitation for $0.5 \mathrm{~h}$. An ultrasonic clean bath apparatus was employed for the MWCNT dispersion. The obtained suspension was kept in resting for $22 \mathrm{~h}$ before submitted to refluxing at $100^{\circ} \mathrm{C}$ for $8.5 \mathrm{~h}$. After refluxing, the suspension was kept in resting again for $16 \mathrm{~h}$ and subsequently, it was washed and centrifuged (at $4000 \mathrm{rpm}$ ) several times using de-ionized water, until a neutral $\mathrm{pH}$ was reached. The functionalized MWCNT (MWCNT-F) was finally dried at $80^{\circ} \mathrm{C}$ for $24 \mathrm{~h}$. For the $\mathrm{Ag}$ decoration in aqueous solution of $\mathrm{AgNO}_{3}$, typically $0.1 \mathrm{~g}$ of MWCNT-F was de-agglomerated in an agate mortar and added to $50 \mathrm{ml}$ of $\mathrm{AgNO}_{3}$ solution (0.1 molar), under ultrasonic agitation for $0.5 \mathrm{~h}$ using an ultrasonic cleaning bath device. The obtained solution was kept in resting for $24 \mathrm{~h}$, and subsequently it was washed in de-ionized water and centrifuged at $4000 \mathrm{rpm}$. Finally, the Ag-decorated MWCNT-F was dried at $50^{\circ} \mathrm{C}$ for $24 \mathrm{~h}$. When comparing to the reference procedure ${ }^{9}$, the Ag-decoration route performed in the present work is simpler, as it does not include a final calcination step at temperatures between 400 and $800^{\circ} \mathrm{C}$ under argon atmosphere.

\subsection{Single step Ag decoration}

Typically, $0.2 \mathrm{~g}$ of MWCNT were de-agglomerated in an agate mortar and dispersed into $260 \mathrm{ml}$ of DMF reductant. $0.2 \mathrm{~g}$ of SDS were also added to this suspension, in order to help on the dispersion. The $\mathrm{pH}$ of the suspension was adjusted to 6 , adding a solution of $\mathrm{HNO}_{3}(0.01 \mathrm{~mol} / \mathrm{l})$. Approximately $25 \mathrm{ml}$ of $\mathrm{HNO}_{3}$ solution were necessary to adjust the ph. This suspension was ultrasonically stirred for $1 \mathrm{~h}$ using an ultrasonic cleaning bath apparatus, and subsequently $60 \mathrm{ml}$ of $\mathrm{AgNO}_{3}$ solution $(0.1 \mathrm{~mol} / \mathrm{l})$ were also added. The suspension was kept between 60 and $62^{\circ} \mathrm{C}$ for $1 \mathrm{~h}$ under magnetic stirring and another $24 \mathrm{~h}$ without heating or agitation. The suspension was finally washed in de-ionized water and centrifuged (at $4000 \mathrm{rpm}$ ) few times, and dried at $50^{\circ} \mathrm{C}$ for $24 \mathrm{~h}$.

\subsection{CNTs characterization}

MWCNT, MWCNT-F and Ag decorated MWCNT-F were characterized by TEM, STEM, Raman and FT-IR spectroscopy. For TEM and STEM analysis the samples were dispersed in acetone under ultrasonic agitation, and captured from the suspension using a carbon film covered copper grid. The samples were observed in a Phillips CM120 TEM and in a FEI TECNAI G ${ }^{2}$ F20 high resolution TEM/STEM microscope, both equipped with energy dispersive spectroscopy microanalysis (EDS). The last was also equipped with a high-angle annular dark field detector, which permits to obtain high-angle annular dark-field images (HAADF). Raman spectroscopy was performed using a micro-Raman Renishaw microscope system 2000, employing an argon laser (514.5 nm wavelength). Samples for FT-IR spectroscopy were mixed and pressed in $\mathrm{KBr}$ pellets, and analyzed in a FT-IR-Affinity-1 (Shimadzu) equipment. $\mathrm{KBr}$ was dried at $100^{\circ} \mathrm{C}$ for $24 \mathrm{~h}$, before preparing the samples.

\section{Results and Discussion}

\subsection{Initial MWCNT characterization}

Figure 1A shows a TEM image, where the typical morphology and the high aspect ratio of the nanotube can be observed. HRTEM image in Figure 1B shows the presence of multiple graphene layers that form the wall of the tube, which has an average diameter of about $10 \mathrm{~nm}$.

\subsection{Two steps Ag decorating:}

STEM analysis performed on MWCNT-F reveals that, after the acid refluxing (first step of the procedure), no 


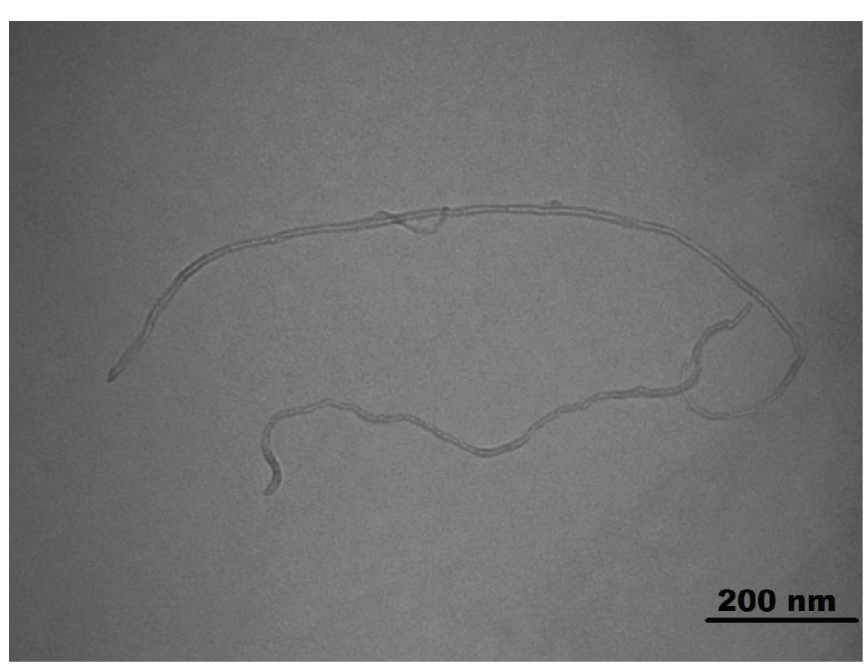

(a)

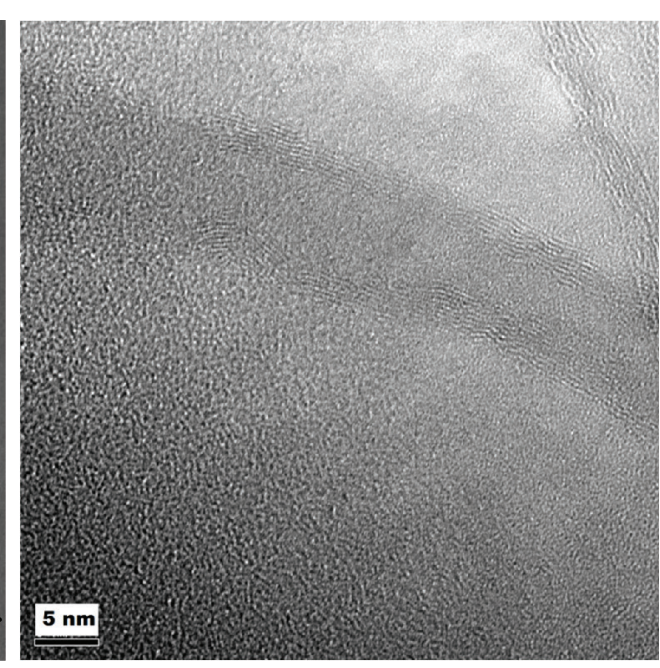

(b)

Figure 1. TEM images of pristine MWCNT used in the present work: A- Low magnification image showing their high aspect ratio: B- High magnification image, showing the graphene layers that form the tube wall.

perceptible changes are observed on their length. The surface is also free of observable defects, as shown in a STEM bright field image (Figure 2).

After the $\mathrm{AgNO}_{3}$ solution treatment, nanoparticles of $\mathrm{Ag}$ were formed and uniformly deposited on the MWCNT-F surface. Figure $3 \mathrm{~A}$ shows these nano-particles of $\mathrm{Ag}$ in a STEM bright field image. Figure 3B shows the same image in STEM dark field mode, where the electrons diffracted by the heavier Ag particles (compared to the MWCNT) are seen with a better contrast. In order to improve the atomic number $(Z)$ contrast, the HAADF mode in the STEM was employed, collecting diffracted electrons at higher angles. The Figure 4A shows in detail the Ag decorated MWCNT surface covered by bright Ag nano-particles, which identification was also confirmed by EDS microanalysis (Figure 4B). The Cu peaks present on the EDS spectrum came from the grid specimen holder.

Structural changes caused by functionalization and $\mathrm{Ag}$ decoration can be observed by changes on Raman spectroscopy bands. The Figure 5 shows the obtained spectra from pristine MWCNT, MWCNT-F and Ag decorated MWCNT-F, where first and second orders D, G and G' bands are seen in all conditions, around their typical values for the incident laser energy employed: 1350, 1580 and $2684 \mathrm{~cm}^{-1}$, respectively. D+G band around $2920 \mathrm{~cm}^{-1}$ is also present in all samples. However, some important differences between the spectra have to be highlighted. The G band intensity seems to decrease, relative to the $\mathrm{D}$ band after refluxing and mainly after $\mathrm{Ag}$ decoration. The same trend related to G' band can be observed. As the D band is frequently related to structural disorders of carbon based materials ${ }^{25,26}$, the $\mathrm{D}$ to $\mathrm{G}$ band intensity ratio (ID/IG) is commonly used to monitor induced defects during nanotubes processing. From Lorentzian fitting of the spectra, we could observe that ID/IG slight increased from 1,05 to 1,17 from pristine to functionalized MWCNT. However, the increase of ID/ IG to 2,90 for Ag-decorated MWCNT was remarkable. A

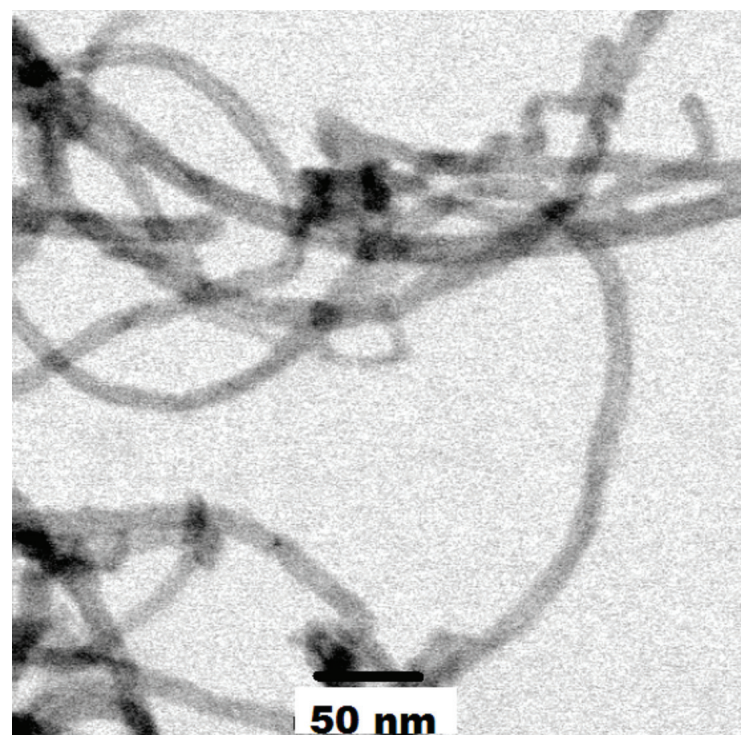

Figure 2. Bright field STEM image of MWCNT-F after acid refluxing, corresponding to the first of the two steps Ag decorating procedure.

closer view of the $\mathrm{G}$ band reveals the presence of a shoulder at the right side of the peak of the pristine spectrum. This shoulder evolves to a more defined peak for functionalized and mainly for Ag decorated MWCNT. This second peak at around $1620 \mathrm{~cm}^{-1}$ can be attributed to the D' band which origin is due to the same double resonance process as the $\mathrm{D}$ and its overtone $\mathrm{G}^{\prime}$ bands ${ }^{25-27}$. The deconvolution of the peak in the range of 1500 to $1750 \mathrm{~cm}^{-1}$ for the functionalized MWCNT, is shown as a detail in Figure 5, as an example.

The ID/IG evolution confirms that some disorder has been introduced to MWCNT due probably to structural modifications at the surface, as they are functionalized and subsequently Ag decorated. It seems that the presence 


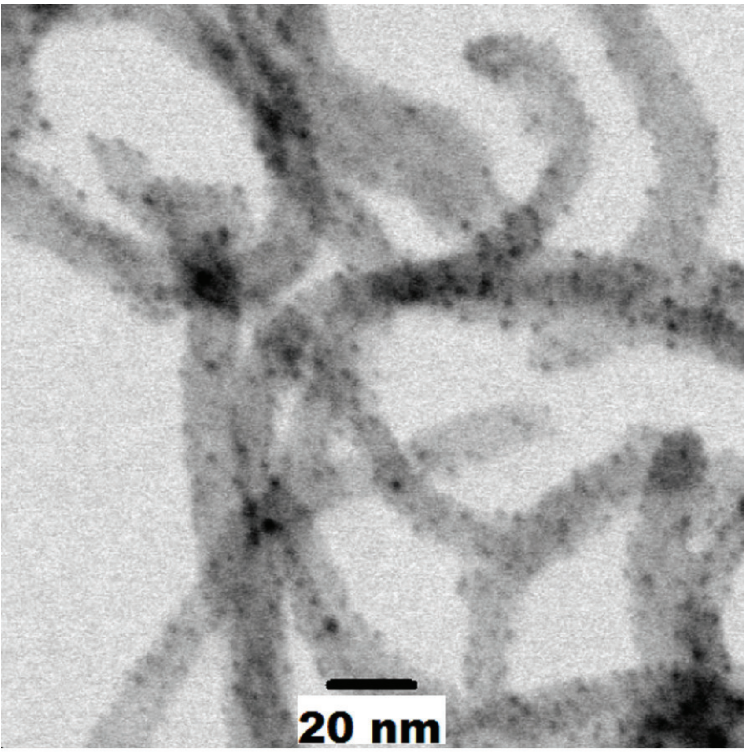

(a)

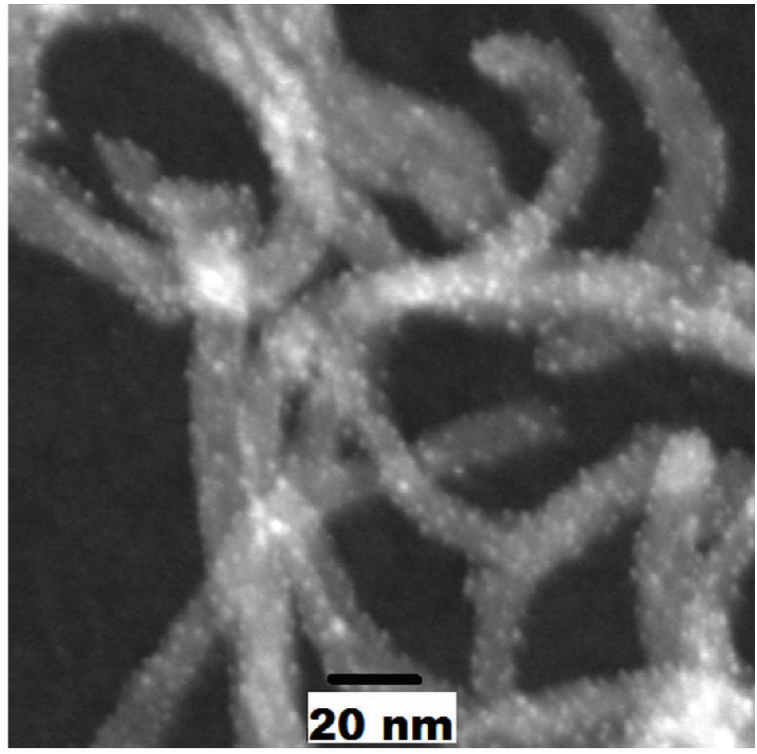

(b)

Figure 3. STEM images of Ag decorated MWCNT-F. 3A shows a STEM bright field image (using transmitted electrons) showing dark Ag nano-particles homogeneously dispersed onto the MWCNT surface. 3B shows the same image in STEM dark field mode, formed by diffracted electrons, where the diffracted electrons collected from Ag particles are bright.

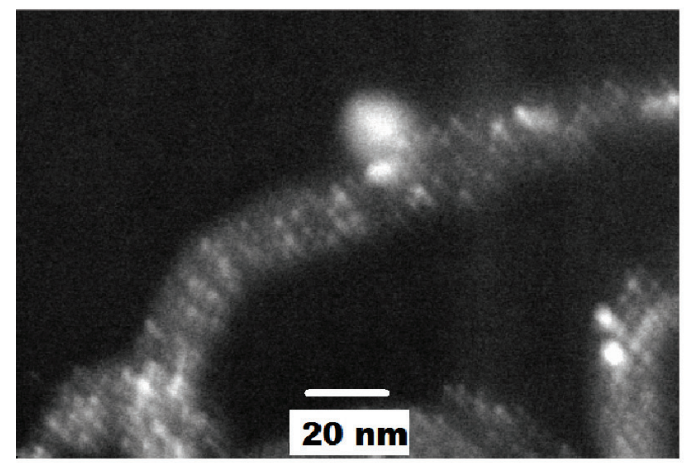

(a)

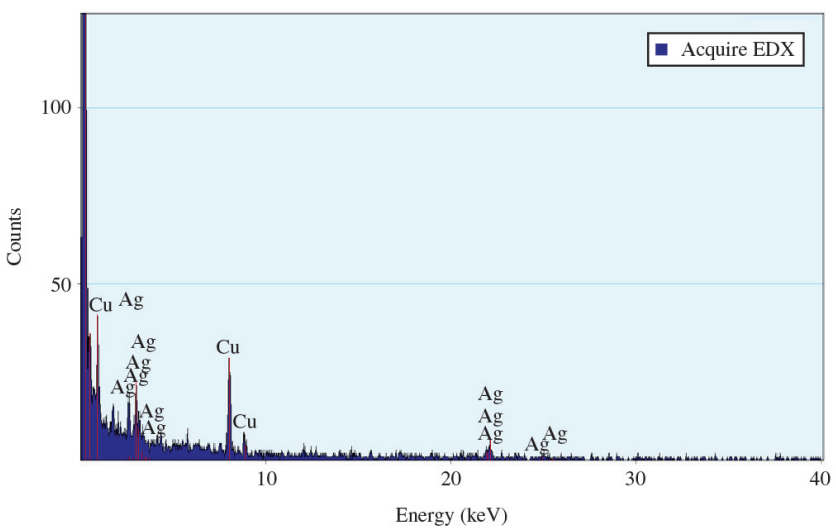

(b)

Figure 4. A- Detail of Ag decorated MWCNT-F surface in HAADF STEM mode. Ag nano-particles, having higher atomic number than carbon, diffracts electrons at higher angles. B- EDS spectrum collected from one of the bright particle in 4A.

of Ag ions on the MWCNT surface causes more evident changes into the Raman scatter intensity. Furthermore, a more defined D' peak in the same processing sequence reinforces this conclusion.

The literature frequently reports symmetrical or asymmetrical peaks broadening or shifting as a result of CNT decoration ${ }^{16,19}$. G peak asymmetrical broadening accompanied by a widening D peak in graphite intercalation by $\mathrm{HNO}_{3}$ has been attributed to the formation of a thin amorphous oxide layer ${ }^{28}$. However, in the present work such features were not observed. In fact, the D' appearance can account for an asymmetric $\mathrm{G}$ peak broadening.

FT-IR analysis was performed in order to identify chemical changes on MWCNT surface resulting from the functionalization step. The spectra obtained for pristine and MWCNT-F are shown in Figure 6. In general, the bands are very small and difficult to distinguish before a careful noise filtering procedure. Typical $\mathrm{C}=\mathrm{C}$ bond stretching are observed on both spectra, at a wavenumber of about $1550 \mathrm{~cm}^{-1}$. It is worth to mention that this value is slight below the typical values of $1570 \mathrm{~cm}^{-1}$, frequently reported $^{9,14,15,19,21,22}$. Other band present in both spectra is around $3450 \mathrm{~cm}^{-1}$ and is probably related to adsorb $\mathrm{O}-\mathrm{H}$ groups from humidity ${ }^{14,15,29,30}$. Three additional bands appear on the MWCNT-F spectrum, at 1170 (strong), 1720 (weak) and $3150 \mathrm{~cm}^{-1}$ (strong). The two first bands can be related to $\mathrm{C}-\mathrm{O}$ and $\mathrm{C}=\mathrm{O}$ bond stretching, respectively, and can be recognized as typical bands resulting from carboxyl 


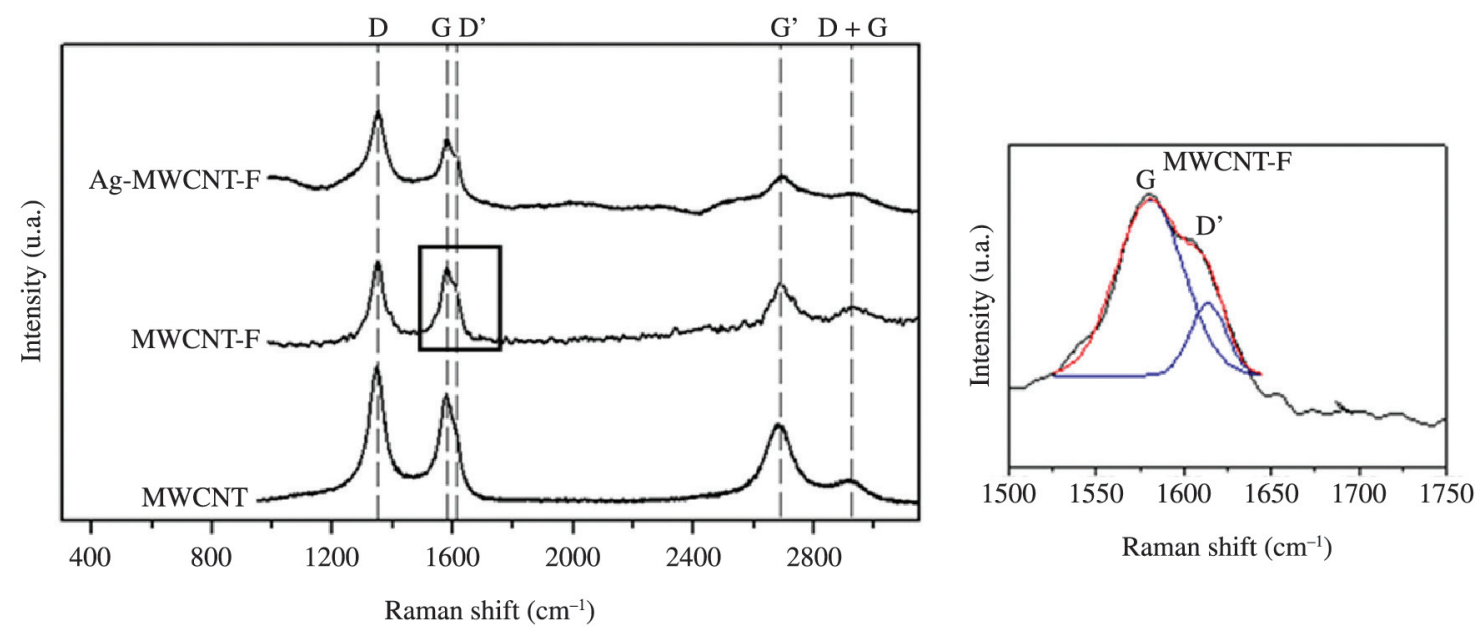

Figure 5. Raman spectroscopy results performed on MWCNT, after each of the two steps Ag decoration procedure. Spectrum from pristine MWCNT is also present, for comparison purpose. Peak deconvolution in the range of $1500-1750 \mathrm{~cm}^{-1}$ is shown in detail, revealing the individual contribution of G and D' intensities in the MWCNT-F spectrum.

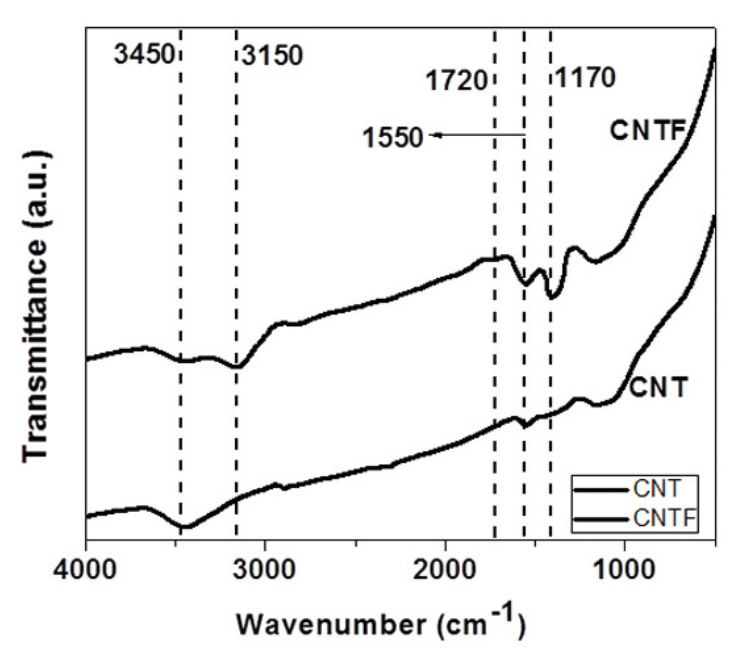

Figure 6. FT-IR spectrum of the MWCNT-F sample, corresponding to the first of the two steps Ag decorating procedure. Pristine MWCNT spectrum is also present, for comparison purpose.

groups $^{8,13,14,20,23,24}$. The origin of the strong band observed at $3150 \mathrm{~cm}^{-1}$ is unclear. This region of the IR spectrum refers typically to $\mathrm{O}-\mathrm{H}$ bond stretching, from adsorbed humidity or even from hydroxyl C-OH groups. As it occurs only for MWCNT-F samples, one could suppose that the band at $3150 \mathrm{~cm}^{-1}$ can be related to hydroxyl groups resulting from the acid functionalization step.

From the results obtained from the two steps $\mathrm{Ag}$ decorating MWCNT process, it can be deduced that the acid refluxing, corresponding to the functionalization step, was efficient in forming functional carboxyl and probably hydroxyl type groups. These functional groups act as nucleation sites for $\mathrm{Ag}$ ions that are in solution, during the second step of decorating procedure. Ag particles of few nanometers homogeneously precipitate at the MWCNT, impregnating their surface. High temperature calcination ${ }^{9}$ wasn't necessary to form these Ag dispersed particles in the present work, although the Ag particles seem to be much bigger in the reference work, apparently resulting from the high temperature treatment. It is supposed that besides Ag, it is also possible to decorate MWCNT with other metallic ions using the same process, if these ions are available in the form of water soluble salts.

\subsection{Single step Ag decorating}

Figures 7A and 7B show a STEM bright field and dark field images (respectively) taken from MWCNT submitted to the single step Ag decoration procedure. Sub-micrometric Ag particles are visible, dark in $7 \mathrm{~A}$ and bright in $7 \mathrm{~B}$, due to the same contrast techniques as in Figure 3. These particles are heterogeneously mixed to the nanotubes and apparently are not strongly attached to them. It is not clear if the $\mathrm{Ag}$ particles in this case contribute to MWCNT agglomeration, as some of them seem to be entrapped inside bundles. It is evident that, under the experimental conditions of this work, the single step Ag decorating procedure was unsuccessful on creating adequate conditions on the MWCNT surface for Ag ions deposition. This result differs from a reference work where Ag nano-particles were homogeneously formed over CNT surface using the same procedure ${ }^{16}$. Surprisingly, Raman spectra analysis in the reference work did not result on ID/IG changes due decoration procedure, and no additional comments are drawn on the presence of $D^{\prime}$ and G' bands, although $G$ band peaks seems not to be sharp at the top. The reference work authors ${ }^{16}$ also argue that a small D band shifting to higher wave numbers suggests a charge-transfer process between Ag particles and MWCNT surface, but they did not mention that $\mathrm{G}$ band did also shifted to higher wave numbers. Experimental details can account for significant differences on results, when an experimental procedure is replicated. In the present work the resting time after stirring was shorter than in the reference work: 24 versus $48 \mathrm{~h}$. However, this difference seems to be improbable to explain the different results, as after $24 \mathrm{~h}$, 


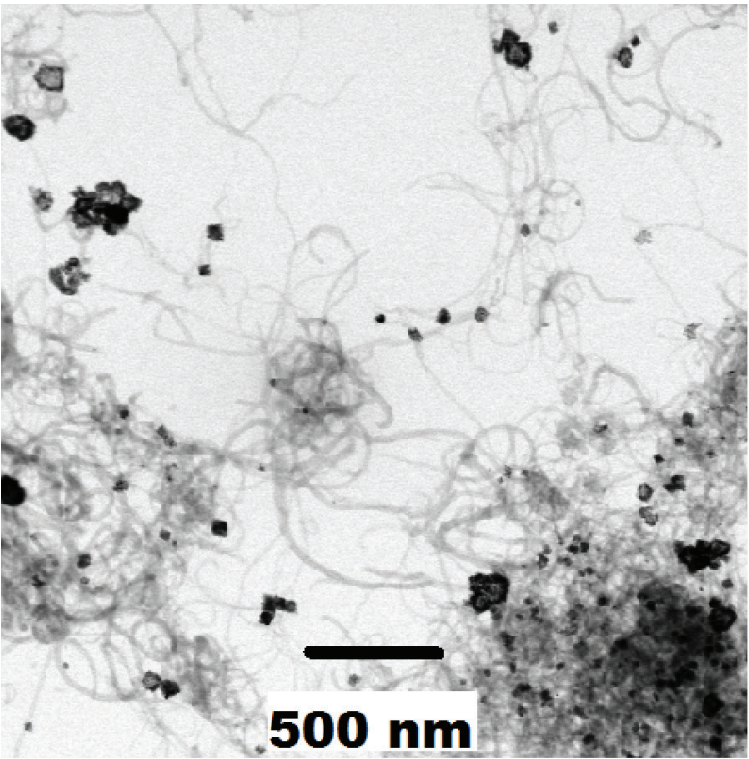

(a)

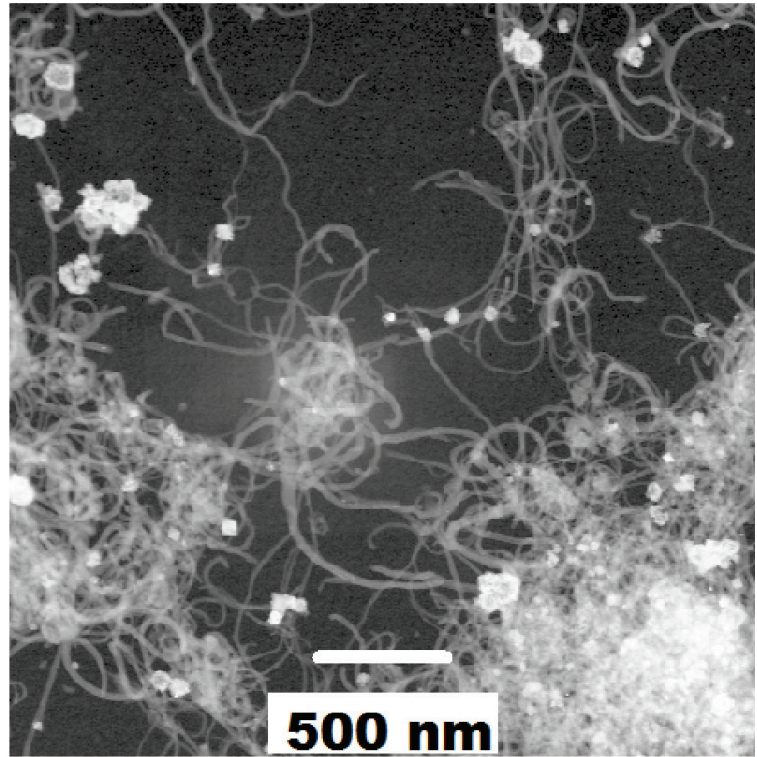

(b)

Figure 7. Aspect of MWCNT after the single step Ag decorating procedure. A- Bright field STEM image shows Ag sub-micrometric particles (dark) heterogeneously mixed to the nanotubes. B- The same image formed by electrons diffracted by Ag particles (dark field image).

the Ag particles were already agglomerated, as observed in Figure 7. The stirring conditions can account for important differences on Ag decoration results. Such conditions are very difficult to quantify for comparison purposes, but it seems that a higher power ultrasonic device could significantly improve the one step deposition process, when comparing to the simple cleaning bath ultrasonic apparatus with subsequent magnetic stirring (during the warm stirring step) used in this work.

\section{Conclusions}

Two different experimental routes for MWCNT surface modification by Ag ions decoration were evaluated: a modified two steps process, based on CNT acid refluxing followed by the suspension in an $\mathrm{AgNO}_{3}$ solution ${ }^{9}$, and a single step process, based on the activation of CNT surface by $n$-dimethylformamide (DMF) in an $\mathrm{AgNO}_{3}$ solution ${ }^{16}$. FT-IR and Raman spectroscopy, as well as TEM, STEM and HRTEM microscopy were employed in order to characterize the products. From the obtained results, the following conclusions can be stated:

- The two steps Ag decorating procedure, consisting of a first acid functionalization followed by a

\section{References}

1. Thostensona ET, Renb Z and Choua TW. Advances in the science and technology of carbon nanotubes and their composites: a review. Composites Science and Technology. 2001; 61(13):1899-1912. http://dx.doi. org/10.1016/S0266-3538(01)00094-X second immersion in an $\mathrm{Ag}$ ion rich solution was successful in producing deposits of Ag nano-particles homogeneously distributed on the MWCNT surface;

- The first acid functionalization step results on the creation of carboxyl and probably hydroxyl groups on the MWCNT surface, which act as sites for Ag ions deposition during the second decoration step. This process is potentially applicable to form deposits of other metallic ions available in the form of water soluble salts;

- The single step Ag decoration procedure was unsuccessful to produce homogeneously dispersed Ag nano-particles over the MWCNT surface. In the present work, Ag forms sub-micrometric particles mixed to the MWCNT, probably due the use of low power stirring conditions.

\section{Acknowledgements}

The authors are very thankful to researchers at Associated Laboratory for Sensors and Materials from the National Institute for Space Research (LAS-INPE - Brazil), for the Raman Spectroscopy analysis.

2. Paradise M and Goswami T. Carbon nanotubes-Production and industrial applications. Materials \& Design. 2007; 28(5):14771489. http://dx.doi.org/10.1016/j.matdes.2006.03.008

3. George R, Kashyap KT, Rahul R and Yamdagni S. Strengthening in carbon nanotube/aluminium (CNT/A1) composites. Scripta Materialia. 2005; 53(10):1159-1163. http://dx.doi. org/10.1016/j.scriptamat.2005.07.022 
4. Esawi AMK and Farag MM. Carbon nanotube reinforced composites: potential and current challenges. Materials \& Design. 2007; 28:2394-2401. http://dx.doi.org/10.1016/j. matdes.2006.09.022

5. Ang LM, Hor TSA, Xu GQ, Tung CH, Zhao SP and Wang JLS. Decoration of activated carbon nanotubes with copper and Nickel. Carbon. 2000; 38(3):363-372. http://dx.doi. org/10.1016/S0008-6223(99)00112-8

6. Chiu PW, Duesberg GS, Dettlaff-Weglikowska U and Roth S. Interconnection of carbon nanotubes by chemical functionalization. Applied Physics Letters. 2002; 80(20):38113813. http://dx.doi.org/10.1063/1.1480487

7. Im $\mathrm{H}$ and $\mathrm{Kim} \mathrm{J}$. Effect of homogeneous $\mathrm{Al}(\mathrm{OH})_{3}$ covered MWCNT addition on the thermal conductivity of $\mathrm{Al}_{2} \mathrm{O}_{3} /$ epoxy-terminated poly(dimethylsiloxane) composites. Journal of Materials Science. 2012; 47(16):6025-6033. http://dx.doi. org/10.1007/s10853-012-6510-3

8. Kam NWS, Jessop TC, Wender PA and Dai H. Nanotube molecular transporters: internalization of carbon nanotubeprotein conjugates into mammalian cells. Journal of the American Chemical Society. 2004; 126(22):6850-6851. http:// dx.doi.org/10.1021/ja0486059

9. Li Z, Fan L, Zhang T and Li K. Facile synthesis of Ag nanoparticles supported on MWCNTs with favorable stability and their bactericidal properties. Journal of Hazardous Materials. 2011; 187(1-3):466-472. http://dx.doi.org/10.1016/j. jhazmat.2011.01.050

10. Liu Y, Tang J, Chen X, Chen W, Pang GKH and Xin JH. A wet-chemical route for the decoration of CNTs with silver nanoparticles. Carbon. 2006; 44(2):381-383. http://dx.doi. org/10.1016/j.carbon.2005.09.006

11. Liu X, Yu L, Liu F, Sheng L, An K, Chen H et al. Preparation of Ag-Fe-decorated single-walled carbon nanotubes by arc discharge and their antibacterial effect. Journal of Materials Science. 2012; 47(16):6086-6094. http://dx.doi.org/10.1007/ s10853-012-6523-y

12. Ma PC, Tang BZ and Kim J-K. Effect of CNT decoration with silver nanoparticles on electrical conductivity of CNT-polymer composites. Carbon. 2008; 46(11):1497-1505. http://dx.doi. org/10.1016/j.carbon.2008.06.048

13. Xu Y-J, Rosa A, Liu X and Su D-S. Characterization and use of functionalized carbon nanotubes for the adsorption of heavy metal anions. New Carbon Materials. 2011;26(1):57-62. http:// dx.doi.org/10.1016/S1872-5805(11)60066-8

14. Martis P, Venugopal BR, Seffer J-F, Delhalle J and Mekhalif Z. Infrared irradiation controlled decoration of multiwalled carbon nanotubes with copper/copper oxide nanocrystals. Acta Materialia. 2011; 59(12):5040-5047. http://dx.doi. org/10.1016/j.actamat.2011.04.061

15. Ramanathan T, Fisher FT, Ruoff RS and Brinson LC. Aminofunctionalized carbon nanotubes for binding to polymers and biological systems. Chemistry Materials. 2005; 17(6):12901295. http://dx.doi.org/10.1021/cm048357f

16. Xin F and Li L. Decoration of carbon nanotubes with silver nanoparticles for advanced CNT/polymer nanocomposites. Composites Part A: Applied Science and Manufacturing. 2011; 42(8):961-967. http://dx.doi. org/10.1016/j.compositesa.2011.03.024

17. Xu ZP, Zeng QH, Lu GQ and Yu AB. Inorganic nanoparticles as carriers for efficient cellular delivery. Chemical Engineering
Science. 2006; 61(3):1027-1040. http://dx.doi.org/10.1016/j. ces.2005.06.019

18. Jiang B, Tian C, Song G, Chang W, Wang G, Wu Q et al. A novel Ag/graphene composite: facile fabrication and enhanced antibacterial properties. Journal of Materials Science. 2013; 48(5):1980-1985. http://dx.doi.org/10.1007/ s10853-012-6964-3

19. Zhang Y, Ma H-L, Peng J, Zhai M and Yu Z-Z. Cr(VI) removal from aqueous solution using chemically reduced and functionalized graphene oxide. Journal of Materials Science. 2013; 48(5):1883-1889. http://dx.doi.org/10.1007/ s10853-012-6951-8

20. Kim I-Y, Lee J-H, Lee G-S, Baik S-H, Kim Y-J and Lee Y-Z. Friction and wear characteristics of the carbon nanotubealuminum composites with different manufacturing conditions. Wear. 2009; 267(1-4):593-598. http://dx.doi.org/10.1016/j. wear.2008.12.096

21. Yuan W, Gu Y and Li L. Green synthesis of graphene/Ag nanocomposites. Applied Surface Science. 2012; 261:753-758. http://dx.doi.org/10.1016/j.apsusc.2012.08.094

22. Ye Y and Guo T. Effect of MWNT electroless Ag plating on field emission properties of MWNT/Ag nanocomposite cathodes. Applied Surface Science. 2013; 264:593-597. http:// dx.doi.org/10.1016/j.apsusc.2012.10.071

23. Nam DH, Cha SI, Lim BK, Park HM, Han DS and Hong SH. Synergistic strengthening by load transfer mechanism and grain refinement of CNT/Al-Cu composites. Carbon. 2012; 50(7):2417-2423. http://dx.doi.org/10.1016/j. carbon.2012.01.058

24. Nakamura J, Matsuda K, Kawabata T, Sato T, Nakamura Y and Ikeno S. Effect of silver on the $\beta$ '-phase in Al-Mg-Si-Ag alloy. Materials Transactions. 2010; 51(2):310-316. http://dx.doi. org/10.2320/matertrans.MC200911

25. Dresselhaus MS, Dresselhaus G, Saito R and Jorio A. Raman spectroscopy of carbon nanotubes. Physics Reports. 2005; 409(2):47-99. http://dx.doi.org/10.1016/j. physrep.2004.10.006

26. Antunes EF, Lobo AO, Corat EJ, Trava-Airoldi VJ, Martin AA and Veríssimo C. Comparative study of first- and secondorder Raman spectra of MWCNT at visible and infrared laser excitation. Carbon. 2006; 44(11):2202-2211. http://dx.doi. org/10.1016/j.carbon.2006.03.003

27. Reich S and Thomsen C. Raman spectroscopy of graphite. Philosophical Transactions of the Royal Society of London A. 2004; 362:2271-2288. http://dx.doi.org/10.1098/ rsta.2004.1454

28. Afanasov IM, Shornikova ON, Kirilenko DA, Vlasov II, Zhang L, Verbeeck J et al. Graphite structural transformations during intercalation by $\mathrm{HNO}_{3}$ and exfoliation. Carbon. 2010; 48(6):1862-1865. http://dx.doi.org/10.1016/j. carbon.2010.01.055

29. Peng J, Qu X, Wei G, Li J and Qiao J. The cutting of MWNTs using gamma radiation in the presence of dilute sulfuric acid. Carbon. 2004; 42(12-13):2741-2744. http://dx.doi. org/10.1016/j.carbon.2004.05.015

30. Gong H, Kim S-T, Lee JD and Yim S. Simple quantification of surface carboxylic acids on chemically oxidized multi-walled carbon nanotubes. Applied Surface Science. 2013; 266:219224. http://dx.doi.org/10.1016/j.apsusc.2012.11.152 